\title{
Giant Spin-Polarization and Magnetic Anisotropy of Nanostructures at Surfaces
}

\author{
H. Brune
}

Institute of the Physics of Nanostructures (IPN),

Ecole Polytechniqe Féd érale de Lausanne (EPFL), CH-1015 Lausanne

We present spin-polarized scanning tunneling microscopy measurements demonstrating spin-polarizations of up to $80 \%$ for Co islands on a $\mathrm{Pt}(111)$ surface and a tunnel magneto resistance of $850 \sim 1 \%$ between the islands and an anti-ferromagnetic Cr-coated W-tip. These values remain constant up to $\pm 0.7 \mathrm{~V}$ bias. We report on the magnetic moments and anisotropy energies of two-dimensional Co islands on $\mathrm{Pt}(111)$ comprising only a few atoms. Our results show the correlation between orbital moments and magneto-crystalline anisotropies and reveal that both properties strongly depend on the lateral atomic coordination. The anisotropy of single adatoms is found to be 200 times the Co hop bulk value. We also present well ordered superlattices of Co islands self-assembled on $\mathrm{Au}(788)$. The particles have uniaxial out-of-plane magnetization and no dipolar interactions. They present a model system for ultra-high density storage media since they have the most uniform anisotropy energies and the highest density of non-interacting particles so far realized.

\section{Spin-polarization in STM-Junctions}

Magnetic random access memories (MRAMs) will possibly replace our current dynamic random access memories (DRAMs) due to their shorter access times and to the fact that they are non-volatile. Depending on cost, they may even replace hard-drives and flash-memories. The MRAM cell consists of a planar tunnel junction between two ferromagnets with its tunnel-magneto-resistance (TMR) being used for readout. The TMR is defined in recent papers as $\left(R_{\mathrm{a}}-R_{\mathrm{p}}\right) / R_{\mathrm{p}}$, with $R_{\mathrm{p}}$ and $R_{\mathrm{a}}$ being the junction resistance for parallel and anti-parallel magnetization of the ferromagnets. One finds $\Delta R / R_{\mathrm{p}}=$ with $\left.P=\left(g^{+}-g^{-}\right) / g^{+}+g^{-}\right)$being the ferromagnets spin polarizations and $g^{+}$and $g^{-}$denoting the density of states for spin up, respectively, spin down electrons at $E_{\mathrm{F}}$. For comparison with TMR values mentioned in early papers we note that $\Delta R / \bar{R}=2 \Delta R /\left(R_{\mathrm{p}}+R_{\mathrm{a}}\right)=2 P_{1} P_{2}$ [1] and $\Delta R / R_{\mathrm{a}}=2 P_{1} P_{2} /\left(1+P_{1} P_{2}\right)[2]$. Note also that appreciable differences between the three definitions occur only at high $P$.

Theory predicts that coherent and state selective tunneling in fully epitaxial junctions may give rise to TMRs of the order of $1000 \%$ [3,4]. Experimental junctions have rapidly improved their TMR over recent years; today they come indeed close to the theoretical maximum. A breakthrough was achieved in 1995 when the TMRs went from former values of a few percent up to $18 \%$ in $\mathrm{Fe} / \mathrm{Al}_{2} \mathrm{O}_{3} / \mathrm{Fe}$ [1] and $12 \%$ in $\mathrm{CoFe} / \mathrm{Al}_{2} \mathrm{O}_{3} / \mathrm{Co}$ tunnel junctions [2] (we refer to 
values at $300 \mathrm{~K}$ ). Another order of magnitude was achieved in 2004, where $188 \%$ were reported for fully epitaxial $\mathrm{Fe} / \mathrm{MgO}(100) / \mathrm{Fe}$ junctions [5], $220 \%$ for polycrystalline $\mathrm{FeCo} / \mathrm{MgO} / \mathrm{FeCoB}$ junctions with (001) texture [6]. Subsequently, $230 \%$ were reported for $\mathrm{CoFeB} / \mathrm{MgO}(100) / \mathrm{CeFeB}$ junctions with polycrystalline ferromagnets facilitating fabrication of many junctions with uniform properties [7]. The highest room-temperature TMR-value to date observed for epitaxial junctions is $410 \%$ and has been measured on $\operatorname{Co}(100) / \mathrm{MgO}(100) / \mathrm{Co}(100)$ junctions [8].

However, these TMR values are restricted to very small bias. They steeply decrease as the voltage is increased to technical useful values $[5,6]$. A second important characteristic for applications is therefore the voltage $V_{1 / 2}$ at which the TMR drops to half of its close to zero bias value. This voltage defines the memory output voltage, $V_{\text {out }}=V_{1 / 2}\left(R_{\mathrm{a}}-R_{\mathrm{p}}\right) / R_{\mathrm{a}}$, which is one of the parameters defining the MRAM density limit. In planar junctions, $V_{1 / 2}$ has been increased from 0.2 [2] to $0.6 \mathrm{~V}$ [7]. Compared to the achieved TMR increase this is moderate improvement and prompts the question how much of the bias dependence is being intrinsic. An intrinsic effect is the variation of the $g$ 's with energy. However, this is expected to show up only beyond $0.5 \mathrm{~V}$ and to give rise to a relatively small decrease up to 1 V. Other effects are interfacial spin-scattering, magnon creation in the oxide, or tunneling via trap states formed by oxide defects [9]. Perfect interfaces and perfect oxides would avoid some of these effects. Which one of them is dominant, and what are the ultimate values of $V_{1 / 2}$ and $V_{\text {out }}$ of a perfect junction, are at present open questions. We briefly note that the so-called zero-bias anomaly usually refers to a TMR-peak observed at low $T$ and at a view milli-volts [10]. This peak is related to Kondo and/or inelastic spin-excitations [11] and has to be discerned from the above discussed TMR decrease at large bias values, which currently affects the operation of planar junctions in MRAMs.

The junction of a spin-polarized STM has controlled interfaces and a clean vacuum barrier. Therefore it promises TMR values closer to the theoretical upper limit and higher stability of these values with increasing bias. Most importantly, however, one of the junction interfaces can be "seen" on the atomic scale, enabling to investigate the causes of performance limits of planar junctions in a systematic way. We present spin polarization and TMR measurements on single planar monodomain islands. TMR values reach up to $850 \%$ for vacuum STM tunnel junctions formed by out-of-plane magnetized ferromagnetic bilayer Co islands on $\mathrm{Pt}(111)(140 \mathrm{~K})$ and anti-ferromagnetic Cr-coated W-tips $(280 \mathrm{~K})$. These values are observed up to biases of $\pm 0.7 \mathrm{~V}$, shifting $V_{12}$ far beyond $1.0 \mathrm{~V}$ [12].

The Co islands shown in Fig. 1a) were created by atomic vapor deposition of 0.40 monolayers (ML, $1 \mathrm{ML}$ being one Co atom per Pt surface atom) on a $\mathrm{Pt}(111)$ substrate held at $130 \mathrm{~K}$ and subsequent annealing to $340 \mathrm{~K}$ [13]. The $-9.4 \%$ misfit between Co and Pt leads to partial dislocations in first layer islands [14]. In the double layer islands imaged here the stress is partly relieved by a moiré structure $[15,16]$. The moiré implies smooth transitions of the Co adsorption sites between three-fold hollow and on-top sites leading to a long-period vertical modulation of the atomic positions reflected in their apparent heights in constant current STM 
a)

b)
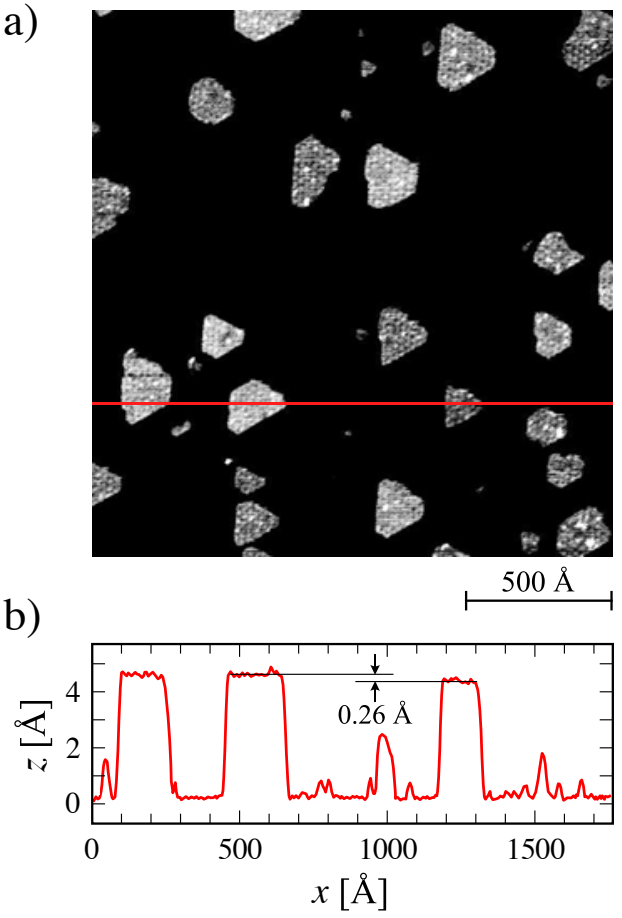

Fig. 1. a) Constant current STM image of double-layer Co islands on Pt(111) recorded with a Cr-coated $\mathrm{W}$ tip. Islands with opposite magnetization appear with two different heights $\left(T_{\text {sample }}=140 \mathrm{~K}, T_{\text {tip }} \approx 280 \mathrm{~K}, V_{\mathrm{t}}=-0.08 \mathrm{~V}, I_{\mathrm{t}}=0.3 \mathrm{nA}\right)$. b) Averaged line-profile ( \pm 5 lines $)$ at the indicated position in figure a) showing a difference of $0.26 \pm 0.05 \AA$ in the apparent height of islands with opposite magnetization.

images. Part of this modulation is still visible in the averaged line scans, and looks as if it was noise (Fig. 1b)).

In addition to the corrugation of the moiré, one clearly discerns two island species by an apparent height difference. This contrast is magnetic since it is only obtained with magnetic tips (either Cr-coated W-tips, or FeMn bulk-tips), and it vanishes above the island blocking temperature of $T_{\mathrm{b}}=180 \mathrm{~K}$, which we independently determined by means of magneto-optical Kerr effect (MOKE) [13]. The MOKE measurements also reveal out-of-plane magnetization in agreement with the fact that spin contrast is only observed with a $\mathrm{Cr}$ coating thickness of 20 $40 \mathrm{ML}$ reported to give out-of-plane polarization of the tip [17].

The magnetic contrast amounts to $\Delta z=0.26 \pm 0.05 \AA$ and can be analyzed in terms of the junction polarization and the TMR that would be observed at constant gap width of the junction. With the above definitions, one finds:

$$
P_{\mathrm{t}} P_{\mathrm{s}}=\frac{I_{\mathrm{p}}-I_{\mathrm{a}}}{I_{\mathrm{p}}+I_{\mathrm{a}}}=\frac{\exp (A \sqrt{\phi} \Delta z)-1}{\exp (A \sqrt{\phi} \Delta z)+1},
$$


where $\phi$ is the average over the work-functions of tip and sample and $\mathrm{A}=$ $2 \sqrt{2 m_{\mathrm{e}} / \hbar^{2}}=1.025 \mathrm{eV}^{-1 / 2} \AA^{-1}$. With a typical value of $\phi=4 \mathrm{eV}$ we find $P_{\mathrm{t}} P_{\mathrm{s}}=$ $0.26 \pm 0.06$ from the difference in apparent height of Fig. 1 . This is significantly higher than $P_{\mathrm{t}} P_{\mathrm{s}}$ values reported between $\operatorname{Co}(0001)$ surfaces and amorphous Cobased alloy tips [9]. Evidently, the spin contrast depends on the orientation of the magnetic moment of the atoms at the apex. These strongly vary for each newly prepared tip, as we conclude from different $\Delta z$ values from day to day, $0.2 \AA$ being a typical value. In a single case we observed a difference of $\Delta z=1.1 \pm 0.1 \AA$, corresponding to $P_{\mathrm{t}} P_{\mathrm{s}}=0.80 \pm 0.04$ [12]. This value implies a polarization of the Co islands of at least $80 \%$, about two times larger than the Co bulk value determined by Andreev reflection [18, 19]. This can be rationalized by the low dimensionality of the islands increasing the density of states at $E_{\mathrm{F}}$, or by state selective tunneling leading to a higher polarization than the state averaged value.

The TMR for a tunnel junction formed by the tip kept at constant height above an island which magnetization switches between up and down evaluates to:

$$
\Delta R / R_{\mathrm{p}}=\frac{2 P_{\mathrm{t}} P_{\mathrm{s}}}{1-P_{\mathrm{t}} P_{\mathrm{s}}}=\exp (A \sqrt{\phi} \Delta z)-1
$$

For our typical $\Delta z$ values Eq. 0.2 gives $\Delta R / R_{\mathrm{p}}=70 \pm 15 \%$, and the largest observed magnetic contrast corresponds to $\Delta R / R_{\mathrm{p}}=850 \pm 200 \%$. The latter value largely overcomes the highest TMR value yet reported and can be considered as bench mark for ideal planar junctions. From density functional theory calculations it is found that adsorbates at the STM-tip apex may cause magnetic contrast in the range of the reported values [20]. We observe stable TMR values up to biases of \pm $0.7 \mathrm{~V}$ [20], which is in agreement with former SP-STM junctions $[9,21]$. Our results suggest that SP-STM experiments are well suited to investigate the effect of structural and chemical defects on the TMR bias dependence.

\section{Magnetic Anisotropy of Single Adatoms}

The energy barrier associated with magnetization reversal by coherent rotation of all spins in a uniaxial system is the magnetic anisotropy energy $K_{2}$, which we call here $K$ for brevity. This energy causes magnetic memories to be non-volatile since it preserves the magnetization from reversing its orientation by thermal excitation. $K$ also defines together with the particle moment $M$ the switching field $H(T)$. The anisotropy energy has several origins, such as shape, magneto-crystalline, surface and interface. In 3D particles several of these causes are present and it is difficult to disentangle them unambiguously. In $2 \mathrm{D}$ nanostructures at single crystal surfaces this is facilitated when morphology and magnetism are investigated insitu on the same sample. This has led to the discovery that the low coordinated step atoms contribute 20 times more to the anisotropy of islands than the laterally 6-fold coordinated atoms sitting inside [13]. 
In Fig. 2 we present the most spectacular example of this coordination effect in showing magnetization curves recorded for single Co atoms adsorbed on a $\mathrm{Pt}(111)$ surface with X-ray magnetic circular dichroism (XMCD) along the easy axis (outof-plane) and at $70^{\circ}$ to it [22]. Isolated Co adatoms have been created by atomic vapor deposition onto a substrate held at low enough temperature to entirely freeze thermally activated tracer diffusion of the deposited species. When atoms adsorb onto a surface, there is the possibility that part of their adsorption energy is transferred into motion along the surface. However, this transient mobility has been found to be absent in all metal/metal systems studied so far [23]. The atoms therefore come to rest at their site of impact. Size distributions for this case called statistical growth can be inferred at low coverage from mathematics [24], and at higher coverages, where deposition onto filled sites comes into play, from kinetic Monte-Carlo or rate theory models [25]. The mean "island" sizes for the coverages used here go from 1.02 (coverage $\theta=0.007 \mathrm{ML}$ ) to 1.11 atoms $(\theta=0.030$ $\mathrm{ML}$ ), therefore at the surface are almost exclusively monomers, in agreement with the uniform apparent height for most of the islands in STM images.

Application of the XMCD sum rules [26] to the spectra measured for the sample shown in Fig. 2a) at saturation and along its easy axis yields $m_{\mathrm{L}}=1.1 \pm 0.1$ $\mu_{\mathrm{B}} /$ atom (the number of core holes needed for this evaluation was calculated

a)

b)
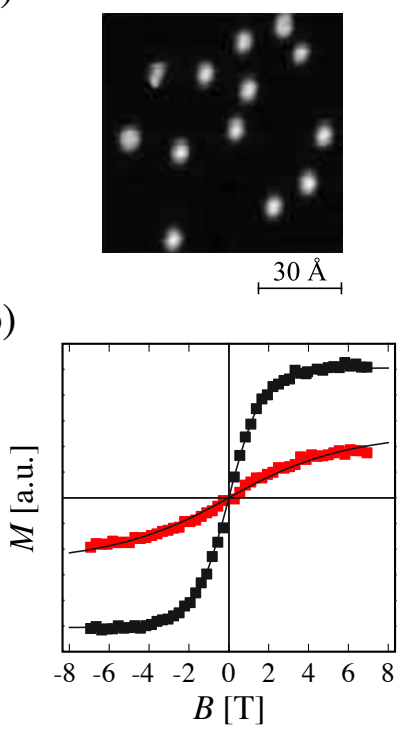

c)
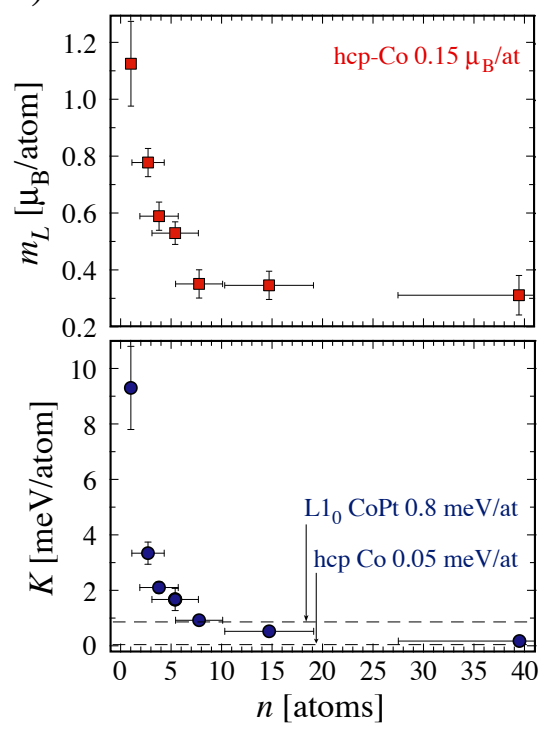

Fig. 2. a) STM image of isolated Co adatoms created by statistical growth on $\operatorname{Pt}(111)(\theta=$ $0.010 \mathrm{ML}$, deposition temperature $T_{\text {dep }}=5.5 \mathrm{~K}$ ). b) XMCD magnetization curves at $0^{\circ}$ (black squares) and $70^{\circ}$ (red squares) with respect to the surface normal measured at $T=$ $5.5 \mathrm{~K}$. The solid lines are fits to the data considering the Zeeman energy resulting from the applied field and the magnetic moments of the atoms and assuming uniaxial out-of-plane anisotropy energy $K$ per atom. c) Atomic orbital moments $m_{\mathrm{L}}$ and anisotropy energies $K$ deduced from XMCD measurements taken out on island ensembles of varying mean size $n$. 
within the local spin density approximation to be 2.4). This value is smaller than $3 \mu_{\mathrm{B}}$ of Co atoms in the gas phase, but much higher than orbital moments in the bulk, where the hybridization of the $d$-states reduces $m_{\mathrm{L}}$ to $0.1-0.2 \mu_{\mathrm{B}}$. The high $m_{\mathrm{L}}$ value comes from the reduced coordination of the adatoms favoring $d$-electron localization and thus the survival of atomic-like character in the $3 d$ orbitals. In a band picture, the $d$-bands are narrowed increasing the local density of states near the Fermi level. This is expected to augment the spin-orbit energy and thereby also the magneto-crystalline anisotropy energy.

Figure $2 b$ ) shows the magnetization along the field applied once parallel to the easy axis (out-of-plane) and once at $70^{\circ}$ to it. The data points represent the peak of the $L_{3} \mathrm{XMCD}$ intensity at $778.6 \mathrm{eV}$ divided by the pre-edge intensity at $775 \mathrm{eV}$ as a function of $B$. The difference between the $0^{\circ}$ and $70^{\circ}$ curves was checked for consistency with the XAS-normalized XMCD spectra. The fits yield $K=9.3 \pm 1.6$ $\mathrm{meV} /$ atom, which is 200 times the Co hop bulk value. It is also much higher than the anisotropies of hard magnets, such as $\mathrm{SmCo}_{5}$ or $\mathrm{CoPt} \mathrm{L}_{0}(K=1.8$, and 0.8 $\mathrm{meV} / \mathrm{Co}$ atom, respectively [27]), and higher than the values formerly reported for atomic Co chains attached to Pt-steps $(K=2.0 \mathrm{meV} / \mathrm{Co}$ atom [28]). The CoPt alloys, the Co chains, as well as the present system, benefit from strong spin-orbit coupling of the Pt $5 d$-states resulting in additional anisotropy energy of the induced magnetization [29]. The fact that the value reported here is much higher than formerly reported ones suggests that coordination has a stronger effect than polarization and spin-orbit coupling of a second element. In line with the dominant role of coordination we observe in Figure 2c) a rapid decease of $m_{\mathrm{L}}$ and $K$ when going from monomers to dimers, trimers, tetramers and so forth [22]. XMCD yields $K=1.0 \pm 0.1 \mathrm{meV} /$ atom for Co heptamers, which are almost entirely composed of step atoms. This is in very good agreement with our MOKEresults on much larger Co islands on $\operatorname{Pt}(111)$, where we found $K_{\mathrm{p}}=0.9 \pm 0.1$ $\mathrm{meV} / \mathrm{step}$-atom [13].

It remains an open issue whether at low $T$ Co monomers on $\operatorname{Pt}(111)$ behave as classical magnetic moments with a remanent magnetization, or whether their ground sate is a superposition of at least two eigenstates of quantum number $+m$ and $-m$. Strong coupling to the surface can favor the first situation, however, experimental evidence for it is currently not available. Single atom experiments exist on systems where the coupling of the magnetic atom to the metal surface has been reduced by an oxide or nitride spacer [30]. In the case of $\mathrm{Fe}$ atoms on $\mathrm{CuN} / \mathrm{Cu}(100)-c(2 \times 2)$ the magnetic state of the atoms is a wavefunction with weight in $m=-2,2$ and 0 , and field dependent spin-excitation spectroscopy measurements with the STM reveal the Hamiltonian containing the anisotropy energies [31]. At which size the transition from quantum mechanical spins to classical magnetic moments with remanent magnetization takes place remains to be investigated. This question is particularly interesting for strongly coupled adsorbate/substrate systems. From our present data, the smallest unit to store information magnetically at room temperature is a ring of 4002 -fold coordinated atoms, each having an anisotropy of $3.3 \mathrm{meV}$. 


\section{Self-assembly of Model Systems for Ultra-high Density Magnetic Storage Media}

Magnetic hard disk media are believed to reach very soon a bit density where the magnetic grains used to store one bit become super-paramagnetic, i.e., their magnetization reverses due to thermal excitation on a time scale shorter than the desired memory retention time of typically 10 years. Since then the memory gets volatile, there is considerable interest in further shifting this limit, and in knowing its ultimate value.

Here we present a way to create model systems suited to explore the ultimate density limit of magnetic information storage. Our example are Co islands selfassembled on a $\mathrm{Au}(788)$ surface. The islands are monodomain particles and have a density of 26 Tbits/in ${ }^{2}$. They are characterized by uni-axial out-of-plane magnetization, by the absence of dipolar interactions, and by unprecedentedly narrow magnetic anisotropy energy and moment distributions [32].

Figure 3a) shows an STM image of a Au(788) surface onto which $0.35 \mathrm{ML}$ of Co have been deposited at $130 \mathrm{~K}$ with subsequent annealing to $300 \mathrm{~K}$. The $\mathrm{Au}(788)$ surface is stable against faceting [33] due to the following reasons. The steps repel each other due to elastic substrate mediated interactions [34]. The sample is cut in such a way that the steps form dense $\{111\}$-facets, which are energetically favored

a)

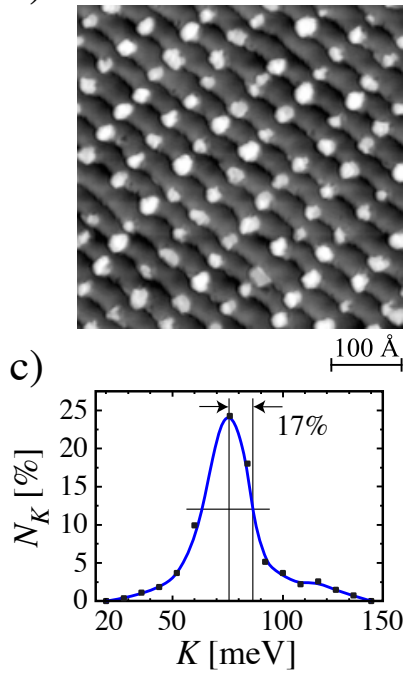

b)

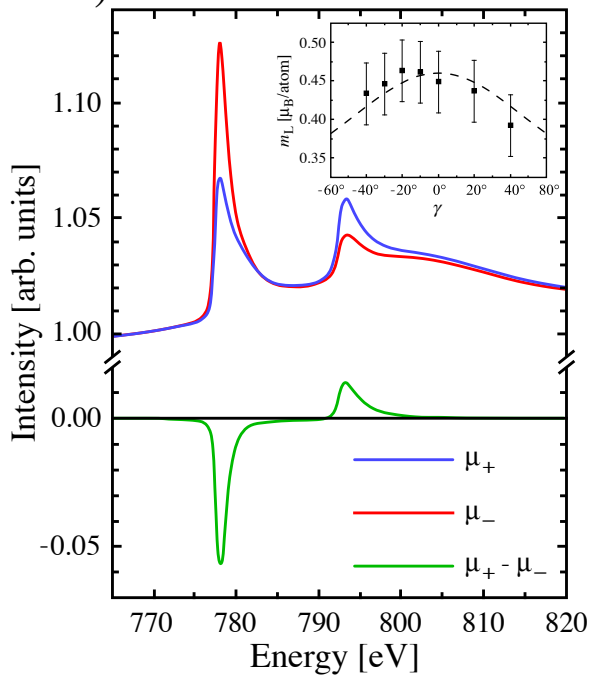

Fig. 3. a) STM image showing Co bilayer islands self-assembled on $\mathrm{Au}(788)$ into a longrange ordered superlattice with a unit-cell of $3.5 \times 7.0 \mathrm{~nm}$, corresponding to a density of 26 Tera-islands $/$ in $^{2}\left(\theta=0.35 \mathrm{ML}, T_{\text {dep }}=130 \mathrm{~K}, T_{\text {ann }}=300 \mathrm{~K}\right)$. b) XAS spectra and the resulting XMCD taken at normal incidence $\left(\gamma=0^{\circ}\right)$ on a sample with $0.35 \mathrm{ML}$ Co $(T=10$ $\mathrm{K}, B=5 \mathrm{~T}, B_{\text {sat }}=2.5 \mathrm{~T}$ ). c) The $K$-distribution inferred from MOKE is with HWHM $=17$ $\%$ roughly two times more narrow than the size-distribution having $\mathrm{HWHM}=32 \%$ $(\theta=0.75 \mathrm{ML}$ deposited in several steps, each time followed by annealing with temperatures as in $\mathbf{a})$ ). 
with respect to the more open $\{100\}$-facets, which would be present on a pristine $\mathrm{Au}(877)$ surface. The $\{111\}$-faceted steps can be crossed perpendicularly [35] by the partial surface dislocations of the $(\sqrt{3} \times 22)$-reconstructed terraces [36]. Finally, elastic interactions align the reconstruction pattern from one terrace to the next. Altogether, this leads to a long range ordered lattice formed by the intersections of steps and reconstruction lines. These intersections are the nucleation sites for Co [33, 37], leading to a regular lattice of double layer high Co islands extending phase-coherently over the entire crystal, since on this surface steps do not destroy the coherence from terrace to terrace.

The XMCD-measurements of the angular dependence of the orbital moment $m_{\mathrm{L}}$ shown in figure $3 \mathrm{~b}$ ) reveal a common easy magnetization axis close to out-ofplane, tilted by $15^{\circ}$ towards the ascending steps. Zero-field susceptibility measurements with MOKE as a function of temperature, $\chi(T)$, show the transition from the blocked to the superparamagnetic state to take place in a narrow temperature window with a width of $15 \mathrm{~K}$. Comparison of STM-derived island size and perimeter length distributions with the $\chi(T)$-curve leads to an anisotropy energy per perimeter atom of $K_{\mathrm{p}}=0.8 \pm 0.1 \mathrm{meV}$ and yields the distribution of island anisotropy energies $K$ shown in Fig. 3c). The $K$-distribution has a $\mathrm{HWHM}_{K}$ of 17 $\%$. This value is almost a factor of two smaller than the one of the size of $32 \%$. In fact, this is expected since the anisotropy is largely given by the perimeter length, which in $2 \mathrm{D}$ has a distribution half as wide as the one of the size. The value of $17 \%$ is also less than half of the best result so far achieved for colloid particles [38]. The fact that our islands have a more narrow distribution of magnetic properties than the much more mono-disperse looking colloid particles can be rationalized by the fact that the magnetic moments of $2 \mathrm{D}$ lattices of colloid particles are not yet uni-axial, implying dipolar interactions. In addition, the competition between several causes of anisotropy, such as faceting, strain or shape anisotropy, may give rise to several easy axes per particle [39].

The value for the anisotropy energy per perimeter atom is in agreement with $0.9 \pm 0.1 \mathrm{meV}$ obtained for $\mathrm{Co} / \mathrm{Pt}(111)$ [13], and with the estimate of $1.0 \pm 0.3$ $\mathrm{meV}$ derived from Ref [40] for Co islands with comparable size on $\mathrm{Au}(111)$. We can compare the anisotropy of the orbital moment, $m_{\mathrm{L}, \|}-m_{\mathrm{L}, \perp}$, derived from $\mathrm{XMCD}$, with the magneto-crystalline anisotropy $K_{\mathrm{MC}}$ per atom, independently derived from MOKE. Both quantities are predicted to be linked to each other by the relation [41]

$$
K_{\mathrm{MC}}=-\alpha \frac{\xi}{4 \mu_{\mathrm{B}}}\left(m_{\mathrm{L}, \|}-m_{\mathrm{L}, \perp}\right),
$$

with the spin-orbit coupling constant $\xi=70 \mathrm{meV}$ for Co [42]. Since MOKE determines the total anisotropy per atom, $K=K_{\mathrm{MC}}+K_{\text {shape }}$, we subtract the shape anisotropy $K_{\text {shape }}=-0.08 \mathrm{meV} /$ atom, obtained assuming circular islands, and obtain $K_{\mathrm{MC}}=0.45 \pm 0.04 \mathrm{meV} /$ atom. With $m_{\mathrm{L}, \|}-m_{\mathrm{L}, \perp}=0.11 \pm 0.01 \mu_{\mathrm{B}}$ we find $\alpha$ $=0.23 \pm 0.02$, confirming previously reported estimates of 0.2 [43]. This result 
points out the direct connection between increased orbital moments of low coordinated atoms and their increased anisotropy energy.

Dipolar interactions between monodomain particles manifest themselves by a flatter than $1 / T$-decrease of the $\chi(T)$-curve above $T_{\mathrm{b}}$ [44]. For the present system, we find the slightly steeper than $1 / T$-decrease, characterizing ensembles of noninteracting particles. The absence of dipolar interactions is further corroborated by a double peak in $\chi(T)$ for a bimodal size distribution, showing that small islands can become superparamagnetic at their blocking temperature, independent of the larger ones which are still blocked and sit next by.

The Co particle superlattices created by self-assembly on a $\mathrm{Au}(788)$ surface have an unprecedented narrow anisotropy distribution, a common out-of-plane easy magnetization axis, and the absence of mutual magnetic interactions at a density record of $26 \mathrm{Tbits} / \mathrm{in}^{2}$. Admittedly, one has to work on the blocking temperature, which is with $50 \mathrm{~K}$ too low. This can be done by adding more Co, for example by growing pillars in the third direction [45], which, according to our data, would still be non-interacting, or by using CoFe or CoPt alloys, which we currently investigate.

The author acknowledges the contributions of his collaborators P. Gambardella, S. Rusponi, T. Cren, N. Weiss, M. Epple, P. Buluschek, and L. Claude to this work, as well as fruitful collaborations with $\mathrm{S}$. Rousset and C. Carbone.

\section{References}

1. T. Miyazaki and N. Tezuka, J. Magn. Magn. Mater. 139, L231 (1995).

2. J.S. Moodera, L. R. Kinder, T. M. Wong, and R. Meservey, Phys. Rev. Lett. 74, 3273 (1995).

3. J. Mathon and A. Umerski, Phys. Rev. B 63, 220403 (2001).

4. X.G. Zhang and W.H. Butler, Phys. Rev. B 70, 172407 (2004).

5. S. Yuasa, T. Nagahama, A. Fukushima, Y. Suzuki, and K. Ando, Nat. Mater. 3, 868 (2004).

6. S.S.P. Parkin, C. Kaiser, A. Panchula, P.M. Rice, B. Hughes, M. Samant, and S.H. Yang, Nat. Mater. 3, 862 (2004).

7. D.D. Djayaprawira, K. Tsunekawa, M. Nagai, H. Maehara, S. Yamagata, N. Watanabe, S. Yuasa, Y. Suzuki, and K. Ando, Appl. Phys. Lett. 86, 092502 (2005).

8. S. Yuasa, A. Fukushima, H. Kubota, Y. Suzuki, and K. Ando, Appl. Phys. Lett. 89, 042505 (2006).

9. W. Wulfhekel, H. F. Ding, and J. Kirschner, J. Magn. Magn. Mater. 242, 47 (2002).

10. M. Jullière, Phys. Lett. A 54, 225 (1975).

11. R. Meservey and P.M. Tedrow, Phys. Rep. 238, 173 (1994).

12. S. Rusponi, N. Weiss, T. Cren, M. Epple, and H. Brune, Appl. Phys. Lett. 87, 162514 (2005).

13. S. Rusponi, T. Cren, N. Weiss, M. Epple, P. Buluschek, L. Claude, and H. Brune, Nat. Mater. 2, 546, 551 (2003).

14. T. Cren, S. Rusponi, N. Weiss, M. Epple, and H. Brune, J. Phys. Chem. B 108, 14685 (2004).

15. P. Grütter and U.T. Dürig, Phys. Rev. B 49, 2021 (1994).

16. E. Lundgren, B. Stanka, M. Schmid, and P. Varga, Phys. Rev. B 62, 2843 (2000).

17. A. Kubetzka, M. Bode, O. Pietzsch, and R. Wiesendanger, Phys. Rev. Lett. 88, 057201 (2002). 
18. R.J. Soulen, J.M. Byers, M.S. Osofsky, B. Nadgorny, T. Ambrose, C.F. Cheng, P.R. Broussard, C.T. Tanaka. J. Nowak, J.S. Moodera, et al., Science 282, 85 (1998).

19. S.K. Upadhyay, A. Palanisami, R.N. Louie, and R.A. Buhrman, Phys. Rev. Lett. 81, 3247 (1998).

20. W.A. Hofer, K. Palotás, S. Rusponi, T. Cren, and H. Brune, Phys. Rev. Lett. 100, 026806 (2008).

21. H.F. Ding, W. Wulfhekel, J. Henk, P. Bruno, and J. Kirschner, Phys. Rev. Lett. 90, $116603(2003)$.

22. P. Gambardella, S. Rusponi, M. Veronese, S.S. Dhesi, C. Grazioli, A. Dallmeyer, I. Cabria, R. Zeller, P.H. Dederichs, K. Kern, et al., Science 300, 1130 (2003).

23. H. Brune, in Physics of Covered Solid Surfaces, edited by H.P. Bonzel (Springer, Berlin, 2001), vol. 42 Subvolume A, Part 1 of Landolt Börnstein New Series, Group III: Condensed Matter, pp. 217-258.

24. M.F. Sykes and M. Glen, J. Phys. A. Math. Gen. 9, 87 (1976).

25. H. Brune, Surf. Sci. Rep. 31, 121 (1998).

26. B.T. Thole, P. Carra, F. Sette, and G.v.d. Laan. Phys. Rev. Lett. 68, 1943 ?1946 (1992).

27. D. Weller and A. Moser, IEEE Trans. Mag. 35, 4423 (1999).

28. P. Gambardella, A. Dallmeyer, K. Maiti, M.C. Malagoli, W. Eberhardt, K. Kern, and C. Carbone, Nature 416, 301 (2002).

29. I.V. Solovyev, P.H. Dederichs, and I. Mertig, Phys. Rev. B 52, 13419 (1995).

30. A.J. Heinrich, J.A. Gupta, C.P. Lutz, and D.M. Eigler, Science 306, 466 (2004).

31. C.F. Hirjibehedin, C.Y. Lin, A.F. Otte, M. Ternes, C.P. Lutz, B.A. Jones, and A.J. Heinrich, Science 317, 1199 (2007).

32. N. Weiss, T. Cren, M. Epple, S. Rusponi, G. Baudot, V. Repain, A. Tejeda, V. Repain, S. Rousset, P. Ohresser, et al., Phys. Rev. Lett. 95.157204 (2005).

33. V. Repain, G. Baudot, H. Ellmer, and S. Rousset, Mat. Sci. Eng. B 96, 178 (2002).

34. G. Prévot and B. Croset, Phys. Rev. Lett. 92, 256104 (2004).

35. V. Repain, J.M. Berroir, S. Rousset, and J. Lecoeur, Europhys. Lett. 47, 435 (1999).

36. J.V. Barth, H. Brune, G. Ertl, and R.J. Behm, Phys. Rev. B 42, 9307 (1990).

37. S. Rohart, G. Baudot, V. Repain. Y. Girard, S. Rousset, H. Bulou, C. Goyhenex, and L. Proville, Surf. Sci. 559, 47 (2004).

38. S.I. Woods, J.R. Kirtley, S. Sun, and R.H. Koch, Phys. Rev. Lett. 87, 137205 (2001).

39. G.A. Held, G. Grinstein, H. Doyle, S. Sun, and C.B. Murray, Phys. Rev. B 64, 012408 (2001).

40. H.A. Dürr, S.S. Dhesi, E. Dudzik, D. Knabben, G.v.d. Laan, J.B. Goedkoop, and F.U. Hillebrecht, Phys. Rev. B 59, R701 (1999).

41. P. Bruno, Phys. Rev. B 39, 865 (1989).

42. T. Koide, H. Miyauchi, J. Okamoto, T. Shidara, A. Fujimori, H. Fukutani, K. Amemiya, H. Takeshita, S. Yuasa, T. Katayama, et al., Phys. Rev. Lett. 87, 257201 (2001).

43. D. Weller, J. Stöhr, R. Nakajima, A. Carl, M.G. Samant, C. Chappert, R. Mégy, P. Beauvillain, P. Veillet, and G. A. Held, Phys. Rev. Lett. 75, 3752 (1995).

44. R.W. Chantrell, N. Walmsley, J. Gore, and M. Maylin, Phys. Rev. B 63, 024410 (2001).

45. O. Fruchart, M. Klaua, J. Barthel, and J. Kirschner, Phys. Rev. Lett. 83, 2769 (1999). 